\title{
A cure for mobility problems
}

\section{As the electronic performance of organic semiconductors and other emergent materials improves, researchers call for attention in extracting charge mobility values from field-effect transistors.}

The art of depositing thin films of various materials onto plastic substrates to create a wide range of flexible optoelectronic devices - from electronic circuits to solar cells, sensors for environmental, robotics and biomedical applications, and bendable displays ${ }^{1}$ - has been a fertile playground for researchers. In fact, several studies have explored the potential of almost all the 'emergent materials' established in the past few decades - including silicon, semiconducting oxides and carbon nanotubes - for realizing flexible circuits with performance comparable with rigid silicon-based microelectronic devices.

The availability of countless strategies to engineer molecular structures with bespoke electronic properties and compatible with solution-based deposition processes makes organic semiconductors one of the most studied families of materials for these applications ${ }^{2}$. All around the world, research groups work on understanding the relationship between the transport of electric charges and the morphology of small molecules and polymers. For instance, they investigate the effects of specific functional groups or of the polymer chain morphology on the way the materials assemble when deposited in a thin film, favouring intermolecular electronic interactions and the flow of electric charges along this conducting layer. Fundamental studies defining general design rules to synthesize high-performance semiconductors or modelling the conduction mechanisms are ongoing, complemented by industry-ready demonstrations of large-scale flexible circuits based on organic transistors fabricated using techniques very similar to those used for printing newspapers.

There is hectic competition between research laboratories to develop semiconductors that can be synthesized and deposited using inexpensive processes, that are robust against mechanical and environmental stress, and that enable the realization of transistors with high performance. A key parameter used to measure and compare the behaviour of different semiconductors in electronic applications is the field-effect mobility, which provides a quantitative indication of the amount of current that can flow in a transistor and of how easy such current can be modulated or switched on and off by changing the voltage applied to the three

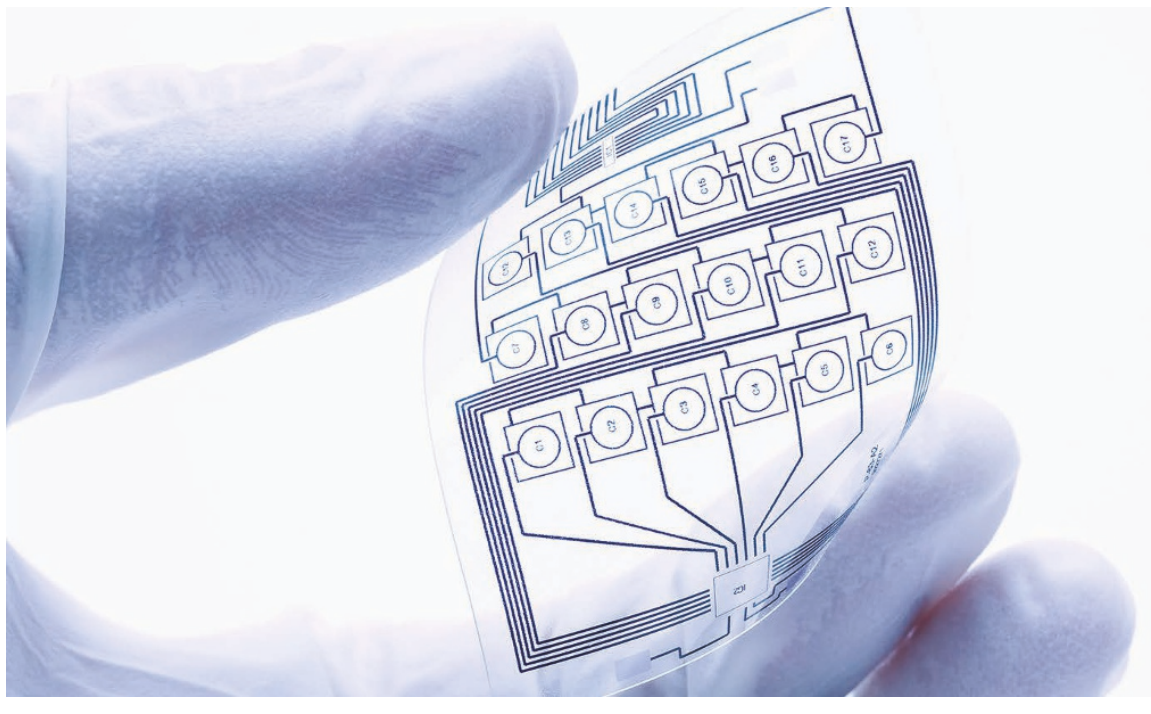

electric contacts of the device. For instance, the maximum operating frequency of an electronic circuit or the maximum sensitivity of a detector depend on this parameter.

Recent literature has, however, shown that there are various ways in which the measurement of the field-effect mobility may go wrong, leading to misrepresentation of the behaviour of the materials and devices studied $^{3}$. In an effort to define guidelines to avoid such pitfalls, a team of experts led by Vitaly Podzorov has described in a Commentary on page 2 some of the challenges and artefacts that can be observed when characterizing organic transistors ${ }^{4}$. They propose, among a list of best-practice rules, the use of a measurement reliability factor - a parameter that measures how distant the behaviour of the characterized device is from that of a textbook field-effect transistor. A value of $100 \%$ would mean that the device follows the ideal Shockley model. Values different from 100\% may hint at nonideal behaviour due to peculiar conduction mechanisms in the materials or device effects not considered in the Shockley model, or at artefacts such as high contact resistance, short-channel effects or wrong estimation of the geometrical parameters of the devices that have altered the mobility estimation. Podzorov and colleagues suggest that, especially in those cases where the reliability factor is very far from $100 \%$ and the claimed field-effect mobility is very high (higher than $5 \mu \mathrm{m}^{2} \mathrm{~V}^{-1} \mathrm{~s}^{-1}$ for organic semiconductors), the device performance - and the mechanisms proposed to explain it - should be confirmed with alternative measurements to avoid data misinterpretation.

As with other similar initiatives ${ }^{5}$, this Commentary aims to give a voice to scientists that want to raise awareness of potential reliability issues of published results, and we hope to trigger a discussion on the best course of action to tackle them. In fact, Nature Materials and the Nature journals have often been a venue to debate methodological problems and bestpractice solutions, and we encourage such contributions (Expert Recommendations ${ }^{6}$ on methodological practices will also be featured systematically in Nature Reviews Physics, launching in 2019). Should you have any relevant comments, feel free to e-mail them to us at materials@nature.com. Supporting opinions, criticisms or suggestions for alternative methodological guidelines to extract mobility values - not limited to transistors based on organic materials but also on other semiconductors, including oxides, hybrid perovskites, 2D materials and nanomaterials - are more than welcome.

\footnotetext{
References

1. Lee, Y. Bloomberg https://bloom.bg/2Bp3eUO (2017).

2. Nat. Mater. 12, 591 (2013).

3. McCulloch, I., Salleo, A. \& Chabinyc, M. Science 352, 1521-1522 (2016).

4. Choi, H. H., Cho, K., Frisbie, C. D., Sirringhaus, H. \& Podzorov, V. Nat. Mater. 17, 2-7 (2018)

5. Nat. Nanotech. 8, 611 (2013).

6. Nat. Rev. Phys. http://go.nature.com/2jlkkwa (2018).
} 